\title{
Photobiomodulation therapy and its effects on muscle damage recovery
}

Dr Jamie Ghigiarelli from Hofstra University investigated the effects of whole-body photobiomodulation (red light) therapy (PBMT)
administered before and after high-intensity training on $12 \mathrm{~m}$ administered before and after high-intensity training on 12 male muscle groups, as handheld devices to treat localised muscle tissues is the conventional method of instrumentation. This study showed no statistical significance but notably there was a $-18 \%$ change for creatinine kinase (CK) levels from $24 h$ hrs to 72 hrs after high-intensity
che training in the difference of average when PBMT group was compared to control conditions.

\section{Lotobiomódulation therapy (PBMT)
is a non-ionising annd non-thermal process used to therapeutica} leviate pain, promote tisșue It works by emitting photons from ear-infrared light $(630-850 \mathrm{~mm})$ on tissues after high-intensity training: The chromophores of the mitochondr

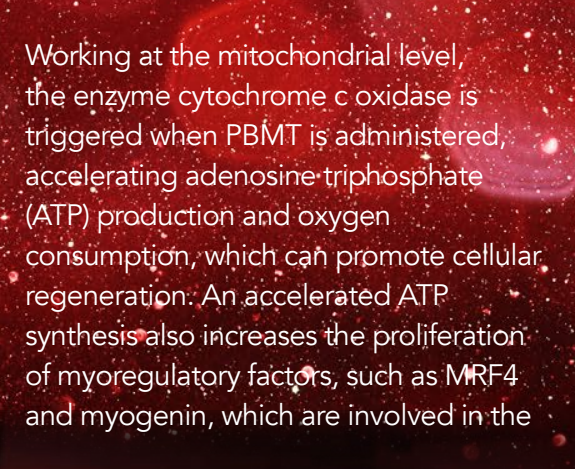

iormation of mature muscle fibres during the muscle repair process.

Through these knóck-on effects, PBMT may minimise muscle damage caused by exercise and improve muscle recovery
especially for hightintensity training athletés. In research literature, PBMT is. allso interchangeably known as.low-leve laser, red light, cold laseir, LED, and n ised to accelerate muscular recovery atter intense exercise include cold-wat
immersion (CWi) and contrast bath. which are commonly compared wit Athletes commonly face muscle fatigue
that typically last for a few days and long
term muscle function impairment âter igh-intensity exercise For ext 13 year-longitudinal. an alysis of European football players found that hamstring injuries are common every season, with one. One to two out of ten injured players also become injured again within two months returning to play. When PBMT were applied before a footbal match, hamstring muscle fatigue was
reduced significantly. Therefore, PBMT is a potential alternative tool to speed up recovery, improve pentormance, and
prevent injuries. prevent injuries.

IS PBMT EFFECTIVE? Other investigations have shown:
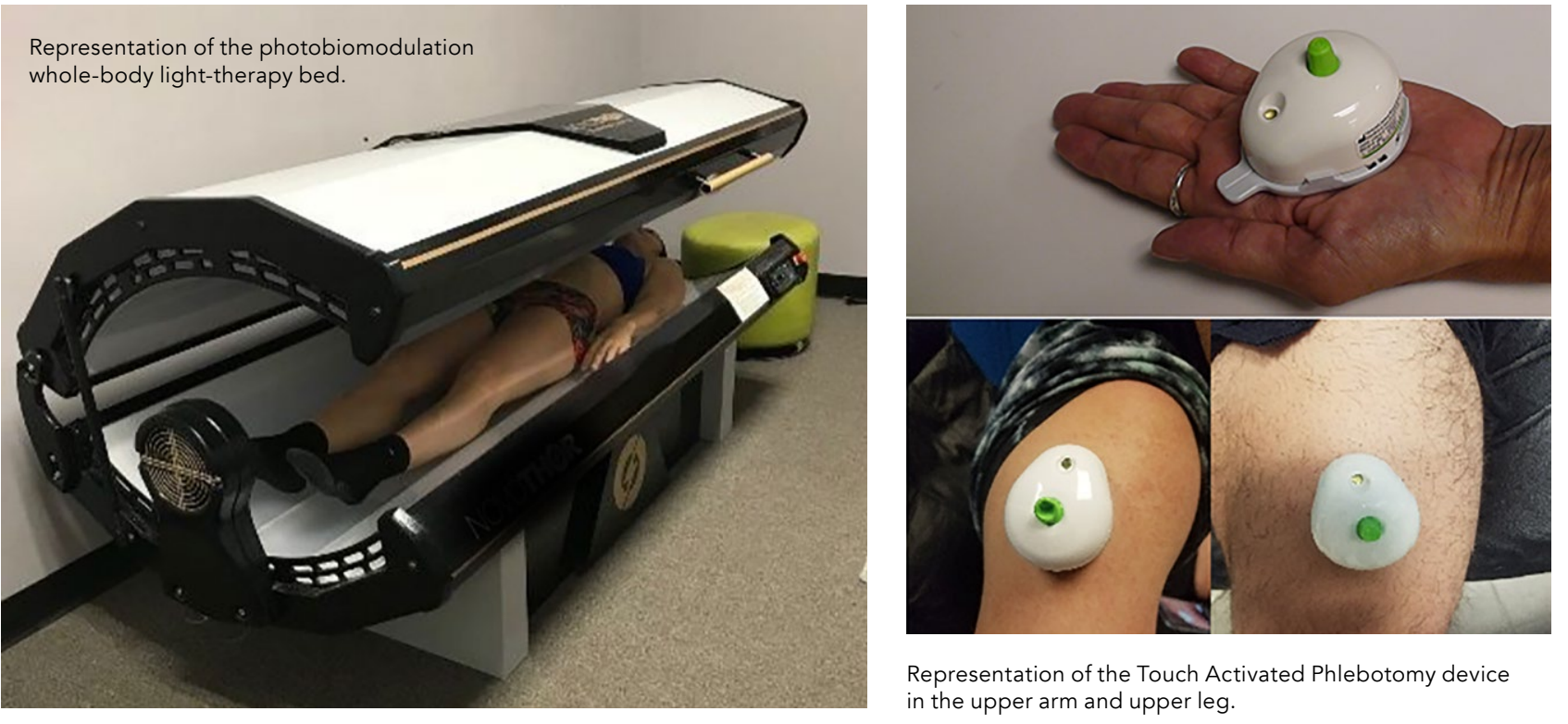

Representation of the Touch Activa
in the upper arm and upper leg.

of PBMT in the management of muscular rehabiltation and peffomance. Some injury time, decreases recovery time between exercise sessions, muscle tiredness and damage, and sleep quality. A number of biochemical markers have been shown to reduce after administration of PBMT, such as cytokine (involved in immune respon to inflammation), blood lactate (associated with low flow of blood and oxygen concentration in cells), and creatine kinase (CK) levels (marker of muscle damage).

density, and energy density. These the dosing of therapy. The lack of parameters means that comparability
with other clinical trials is difficult to determine the effectiveness of PBMT. Therefore, standardisation of exercise protocols and PBMT parameters are

muscle groups in athletic populations and tests biochemical markers. Dr from Hofstra University, which includes Dr Andras Fulop, Dr Katie Sell, and Dr Adam Gonzalez, aimed to circumvent this limitation by investigating the effects of pre-exercise and post-exercise whole-

PBMT is a potential alternative tool to speed up recovery, improve performance, and prevent injuries.

On the other hand, there have been eports that PBMT does not prevent knee arthritis and may not be significantly distinct from placebo on muscular furtherfound that PBMT did not have any significant effect on inflammation, and delayed onset mase soreness after high intensity sprint sessions when compared to placebo and other therapies, such as $\mathrm{CWl}$ and active recovery (AR, which involves individuals performing low-intensity workout after a high-intensity exercise). A 2020 review further found that PBMT is ineffective reducing $\mathrm{CK}$ levels in general exercise (whole body), as compared to highintensity localised exercise.

The differences in research findings can be attributed to the inconsistencies in clinical trials methodology and lack of reporting of PBMT device's technical
parameters, such as wavelength, power needed to improve the use of PBMT in

needed to imprical settings

The differences in results from multiple studies call for further investigation, recommendations given barameter et al (2019) to elucidate the effects of PBMT. There has not been astudy that investigates the effect of PBMT on larger

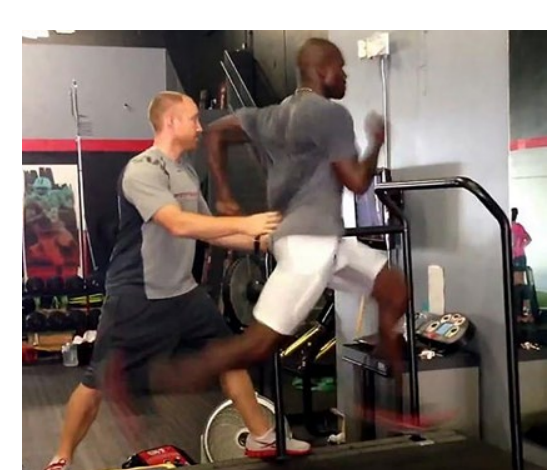

The study investigated the effects of whole.
body $B$ BMMT following treadmill sprints and body PBMT on CK and salivary IL-6 levels in males who performed high-intensity resistance training (identified as the contraction. Blood creatine kinse (CK) is a muscle damage marker and can be used to indicate muscle integrity and an individual's recovery process. Highintensity exercise can cause changes in muscle fibre function and local inflammatory response, whereas low to moderate-intensity exercise have been thown to cause little changes in muscle held device is used to target a specific muscle area, whereas a light bed can be a light bed can potentially target more tissue in a short span of time. Thus, investigating the effects of whole-body
Salivary interleukin-6 (IL-6) is a first "myokine", which is a cytokine applied to the whole body. Therefore, 


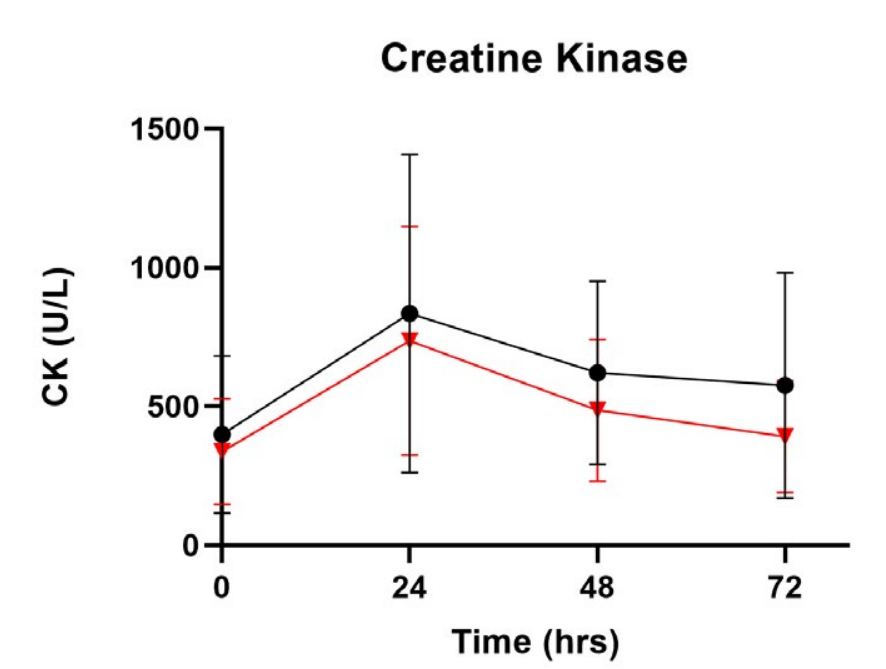

$\bullet$ control

$\rightarrow$ PBMT IL-6 levels were lower in control groups compared participants had lower $\mathrm{CK}$ levels during the PBMT sessions compared to control occasions, whereas five participants had lower CK levels during control. occasions. However, a notable result was that the difference between the average was $-18 \%$ for CK change from 24hrs to 72 hrs after high intensity exercise when comparing PBMT to control conditions.

Even though the experiments do not show positive CK level reduction after administering PBMT, it supports a previous 2020 review that reported PBM is ineffective in reducing CK levels for general exercise compared to localised exercise, and another recent study investigating full body PBMT that showed only a positive efect for LDH (reduced that there is more considerable muscle damage when conducting localised exercise, so PBMT may exert more positive recovery response to reduce $\mathrm{CK}$ levels. In contrast, general or whole-body exercise does not cause considerable stress to have a recovery response, especially because trained participants may already be accustomed to the stress of the exercise programme.

This experiment was conducted over a short span of time with male participants, which limits the generalisability and reproducibility of the investigation. Therefore, in future expentists, both male and female participants could be and PBMT administration could be conducted over a long period of time to possibly yield statistical significance. A variability of age, body composition, and training status could be employed in the methodology of experiments, as these factors are known to account for a CK response from exercise.

In conclusion, this investigation adds to the decade-long research on PBMT and its effects on performance and rehabilitation. The lack of evidence for the use of whole-body PBMT machines require more research such as this. Furthermore, PBMT parameters must be consistent across different be compared.

\section{Behind the Research Q.
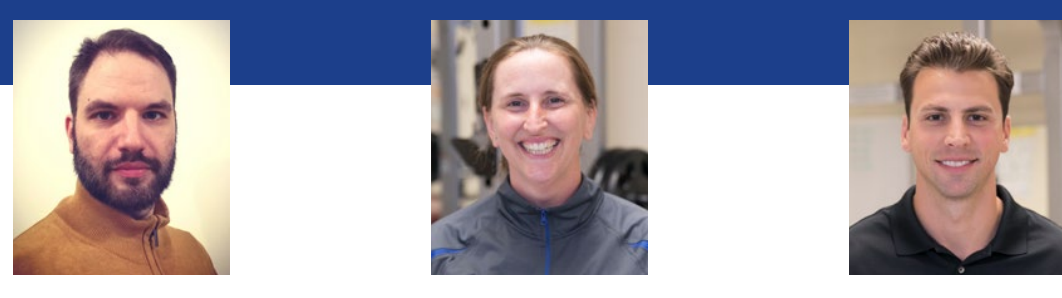

Dr Jamie Ghigiarelli Dr Andras Fulop

E: Jamie.ghigiarelli@hofstra.edu T: +1 516-463-5122

\section{Research Objectives}

Dr Ghigiarelli's primary areas of research are biomechanics, exercise physiology, muscle physiology, sport performance,

\section{Detail}

\section{Address}

Hofstra University, 220 Hempstead Turnpike
115 Hofstra Dome, Hempstead, NY, 11549. USA

Bio

Dr Jamie Ghigiarelli holds a Ph.D. in Exercise Physiology from the University of Pittsburgh. He is an Associate progsom in Sports Science. His primary research aduate program in Sports Science. His primary

Andras Fulop, Sci.D., DPT is a Physical Therapist, and Professor of Human Biology and Sports Sciences.

Dr Katie Sell holds a Ph.D. in Exercise Physiology from the University of Utah. She is a Professor for Hofstra University
and directs the undergraduate Exercise Science program.

Dr Adam Gonzalez holds a Ph.D. in Exercise Physiology from the University of Central Florida. He is an Assistant
Professor for Hofstra University in both the undergraduat and graduate programs.

\section{Collaborators}

- Dr Andras Fulop

- Dr Adam Gonzalez

- Anthony Ferrara

- Luke Pelton

- Shaquille Coke

- Dennis Marshall

- Seventh Sense Biosystems (http://www.7sbio.com/tap)

\section{办}

HOFSTRA
Dr Katie Sell

Dr Adam Gonzalez

\section{References}

- De Marchi, et al. (2019). Photobiomodulation therapy before futsal matches improves the staying time of athletes in the Science. 34 (1), 139-148. - Dornelles, M. P., et al. (2019). Photobiomodulation therapy as a tool to prevent hamstring strain injuries by reducing soccer-
induced fatigue on hamstring muscles. Lasers in Medical induced fatigue on hamstring
Science. $34(6), 1177-1184$. - Ghigiarelli, $J$. J., et al. (2020). The effects of whole-body
photobiomodulation light-bed therapy on creatine kinase and salivary interleukin-6 in a sample of trained males:

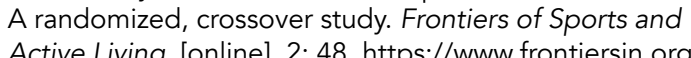
articles/10.3389/fspor2020 00048/full/. B. Junior, E. C., Lopes-Martins, R. A. B. of photobiomodulation therapy in exercise performance and future directions. Brazilian Journal of Physical Therapy. 23 (1), 71-75. - Machado, A. F., et al. (2020). Phototherapy on management of
creatine kinase activity in general versus localized exercise: A creatine kinase activity
systematic review and meta-analysis. Clinical Journal of Sport Medicine. 30 (3), 267-274. - Malta, E. S., et al. (2019). Photobiomodulation by LED does not
alter muscle recovery indicators and presents similar outcomes to cold-water immersion and active
Physiology. 9, 1948. Preliminary Results. Photobiomodulation, Photomedicine, and
Laser Surgery. 38:12:766-772. DOI: 10.1089/photob.2020.4803.

\section{Personal Response} further studies to circumvent the limitations posed by

II Our laboratory currently has a temporary hold on ongoing research projects until our University loosens some of the these limitations, I believe you need to change your subject recruitment and data collection methods. Recently, we
completed a survey study on female martial arts fighters and completed a survey study on female martial arts fighters and
are preparing it for journal submission. We recruited subjects via social media and collected our data through an online survey. In this climate, surveys are ideal because you don't are typical for sports science research. (2019). Clinical and scientific recommendations for the use alter muscle recovery indicators and presents similar or in -Zagatto, A. M., et al. (2020). Full Body Photob Wadulation
Therapy to Induce Faster Muscle Recovery in Water Polo Athletes:

What measures did you have to bring into place for
UNIVERSITY. 